OPEN ACCESS

Edited by:

M. Pilar Francino,

FISABIO - Public Health, Spain

Reviewed by:

Franck Carbonero,

University of Arkansas, United States

Elisabeth Margaretha Bik,

uBiome, United States

${ }^{*}$ Correspondence:

Chitra Dutta

cdutta@iicb.res.in

cdutta.iicb@gmail.com

${ }^{\dagger}$ Present Address:

Chitra Dutta,

Golepark Co-operative Housing

Society, Kolkata, India

Specialty section:

This article was submitted to

Microbial Symbioses,

a section of the journal

Frontiers in Microbiology

Received: 18 October 2016

Accepted: 07 June 2017

Published: 23 June 2017

Citation:

Gupta VK, Paul S and Dutta C (2017)

Geography, Ethnicity or

Subsistence-Specific Variations in Human Microbiome Composition and

Diversity. Front. Microbiol. 8:1162. doi: 10.3389/fmicb.2017.01162

\section{Geography, Ethnicity or Subsistence-Specific Variations in Human Microbiome Composition and Diversity}

\author{
Vinod K. Gupta ${ }^{1,2}$, Sandip Paul ${ }^{1}$ and Chitra Dutta ${ }^{1,2 * t}$ \\ ${ }^{1}$ Structural Biology and Bioinformatics Division, CSIR-Indian Institute of Chemical Biology, Kolkata, India, ${ }^{2}$ Academy of \\ Scientific and Innovative Research, Kolkata, India
}

One of the fundamental issues in the microbiome research is characterization of the healthy human microbiota. Recent studies have elucidated substantial divergences in the microbiome structure between healthy individuals from different race and ethnicity. This review provides a comprehensive account of such geography, ethnicity or life-style-specific variations in healthy microbiome at five major body habitats-Gut, Oral-cavity, Respiratory Tract, Skin, and Urogenital Tract (UGT). The review focuses on the general trend in the human microbiome evolution - a gradual transition in the gross compositional structure along with a continual decrease in diversity of the microbiome, especially of the gut microbiome, as the human populations passed through three stages of subsistence like foraging, rural farming and industrialized urban western life. In general, gut microbiome of the hunter-gatherer populations is highly abundant with Prevotella, Proteobacteria, Spirochaetes, Clostridiales, Ruminobacter etc., while those of the urban communities are often enriched in Bacteroides, Bifidobacterium, and Firmicutes. The oral and skin microbiome are the next most diverse among different populations, while respiratory tract and UGT microbiome show lesser variations. Higher microbiome diversity is observed for oral-cavity in hunter-gatherer group with higher prevalence of Haemophilus than agricultural group. In case of skin microbiome, rural and urban Chinese populations show variation in abundance of Trabulsiella and Propionibacterium. On the basis of published data, we have characterized the core microbiota-the set of genera commonly found in all populations, irrespective of their geographic locations, ethnicity or mode of subsistence. We have also identified the major factors responsible for geography-based alterations in microbiota; though it is not yet clear which factor plays a dominant role in shaping the microbiome-nature or nurture, host genetics or his environment. Some of the geographical/racial variations in microbiome structure have been attributed to differences in host genetics and innate/adaptive immunity, while in many other cases, cultural/behavioral features like diet, hygiene, parasitic load, 
environmental exposure etc. overshadow genetics. The ethnicity or population-specific variations in human microbiome composition, as reviewed in this report, question the universality of the microbiome-based therapeutic strategies and recommend for geographically tailored community-scale approaches to microbiome engineering.

Keywords: body habitats, host genetics, lifestyle, disease susceptibility, non-western people, hunter-gatherers, rural community, urban life

\section{INTRODUCTION}

We share our body space with around 100 trillion microorganisms, collectively known as microbiota (Turnbaugh et al., 2007; Consortium, 2012). The growing perception that our genetic landscape is a summation of the genes embedded in our own genome as well as in genomes of our microbiota (the microbiome), and that our metabolic features present an assemblage of human and microbial traits has led to the launching of numerous microbiome projects worldwide. Recent advancement of culture-independent, high throughput next generation sequencing technologies has enhanced our ability to characterize the human microbiome at various states of health and disease (Turnbaugh et al., 2007; Consortium, 2012). Large-scale endeavors such as the Human Microbiome Project (HMP) have been initiated for characterization of healthy human microbiome (Turnbaugh et al., 2007). Studies are being conducted to explore the plausible disease links of microbiome and efforts are being made to understand how microbiome varies with host lifestyle, genetics, age, nutrition, medication, and environment (Turnbaugh et al., 2006; Blaser et al., 2008; Gao et al., 2008; Islami and Kamangar, 2008; Garrett et al., 2010; Tana et al., 2010; Castellarin et al., 2011; Wang et al., 2011; Kostic et al., 2012; Li et al., 2014; Blekhman et al., 2015; O'Keefe et al., 2015; Falony et al., 2016; Goodrich et al., 2016; Zhernakova et al., 2016).

If we think globally the human microbiome studies are partial, representing for the most part from US, Europe and other so-called WEIRD countries (i.e., Western, Educated, Industrialized, Rich, and Democratic countries) which generally represent urban population (Morton et al., 2015). Only recently, some national and international initiatives have been taken for characterization of human microbiome in diverse ethnic populations and there is a fast growing collection of data describing the microbiome structures in various non-US or nonWestern populations (Figure 1) (Moossavi, 2014). These studies have shown significant variations in microbiome composition in healthy individuals from different race and ethnicity categories (Nam et al., 2011; Nasidze et al., 2011; Yap et al., 2011; Yatsunenko et al., 2012; Mason et al., 2013; Li et al., 2014; Schnorr et al.,

Abbreviations: UGT, Urogenital tract; HMP, Human Microbiome Project; WEIRD, Western, Educated, Industrialized, Rich and Democratic countries; GM, Gut Microbiota; IBD, Inflammatory bowel Disease; AAs, African Americans; NAs, Native Africans; T2D, Type-2 Diabetes; NAFLD, Non-alcoholic fatty liver disease; CCHC, Cameron County Hispanic Cohort; OTU, Operational Taxonomic Unit; URT, Upper respiratory Tract; LRT, Lower Respiratory Tract; BVAB1, Bacterial Vaginosis-Associated Bacterium-1; GIT, Gastrointestinal Tract; PCA, Principal Component Analysis.
2014; Leung et al., 2015; Obregon-Tito et al., 2015; Van Treuren et al., 2015; Gomez et al., 2016). Between-group differences in susceptibilities to many health conditions from preterm birth to type 2 diabetes, obesity and even cancer are being linked to microbiome diversity (Peek and Blaser, 2002; Ley et al., 2005; Turnbaugh et al., 2006; Blaser et al., 2008; Gao et al., 2008; Islami and Kamangar, 2008; Garrett et al., 2010; Tana et al., 2010; Castellarin et al., 2011; Wang et al., 2011; Kostic et al., 2012; Blekhman et al., 2015; O'Keefe et al., 2015; Goodrich et al., 2016). It is an established fact that microbiome composition is linked with various diseases, which motivates the scientific community to identify the microbiome based biomarkers for diagnostic and clinical purposes, but population based variation in microbiome composition between healthy individuals makes it difficult. In case of gut microbiome Falony et al. reported a decrease in the number of core genera from 17 to 14 when they analyzed the gut microbiome data from the populations of Papua New Guinea, Peru, and Tanzania with that of a western dataset including data from Flemish and Dutch cohorts, as well as from UK and US populations (Falony et al., 2016). Population based variation in microbiome profile depends on various population based factors for example, Dutch people consume high milk and low antibiotics compared to other populations of Europe (Zhernakova et al., 2016). So population based variation in microbial profile in healthy individuals must be considered to identify the microbiome based biomarkers for particular diseases. In recent times, a number of reviews have been published summarizing microbiome research from various perspectives, but a comprehensive account of the observations made on geography, ethnicity or life-style-specific variations in microbiome composition is long overdue. The present review attempts to address this issue. It will discuss the major findings on cross-population variations in microbiome composition of various biogeographic spaces considering the five major human body habitats-Oral cavity, Respiratory Tract, Gut, Urogenital Tract (UGT) and Skin. The present article also aims to characterize the geographical-core (present in all populations under the study) microbiota at different body habitats of human (Tables S1-S3). Some recent studies attributed variations in microbiome profiles to methodological biases (DNA extraction, primer choice and amplification methods) (Brooks et al., 2015; Walker et al., 2015; Gerasimidis et al., 2016; Vebo et al., 2016). In the present review, most of the included studies are similar in methodology and despite such similarities in methodology, significant variations were observed in the microbiome profiles of different populations. Thus the methodological biases, if any, could not affect the observations made in this review (Table S4). Geography represents an ensemble of genetic, environmental 


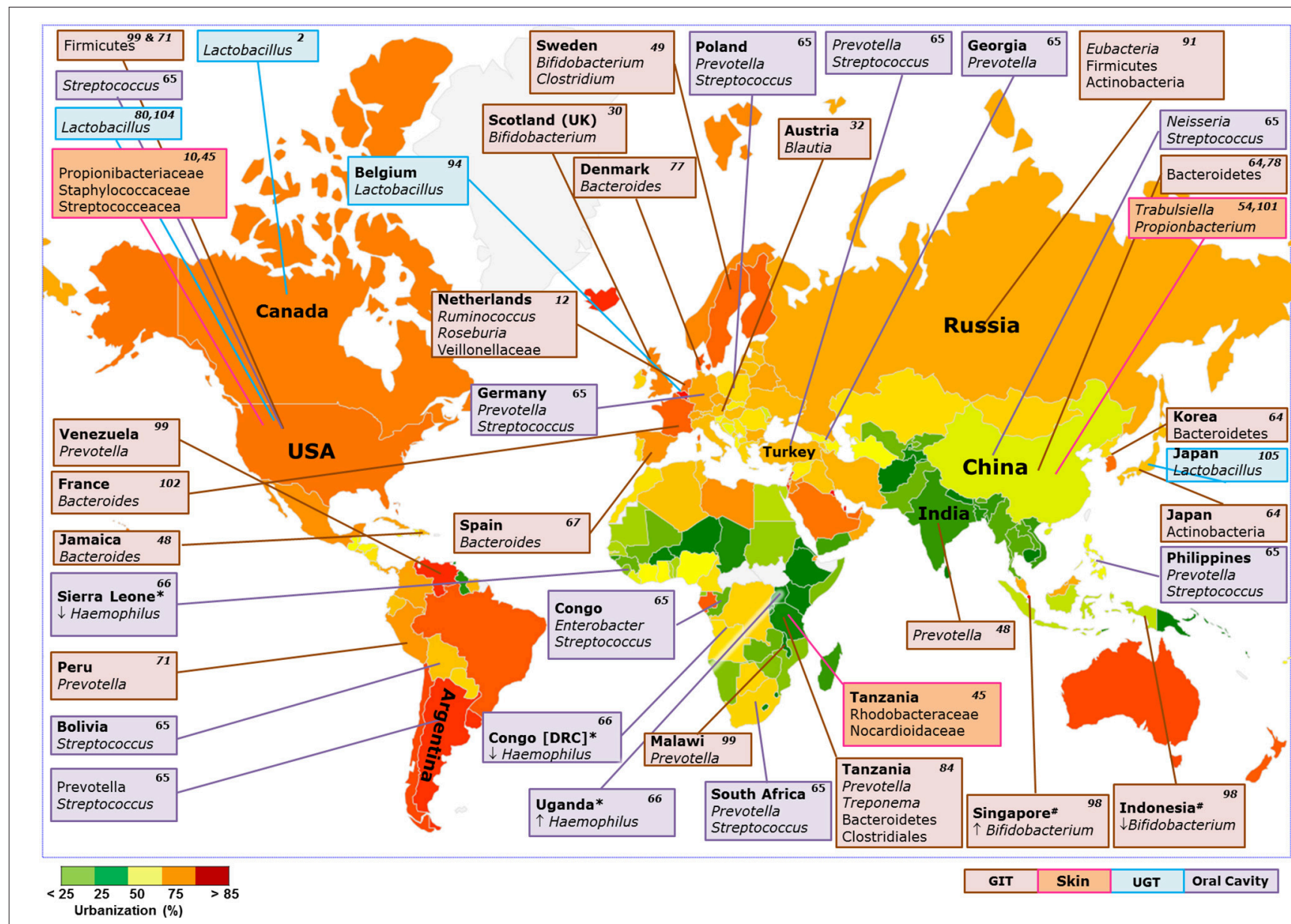

FIGURE 1 | Enriched taxa at various niches of the human body in diverse populations around the world. Box color: body niche; Color in Map: percentage urbanization of countries (http://www.unicef.org/); Up arrow: Dominant abundance of Phylum/Genus compared to respective population; Down arrow: Low abundances of Phylum/Genus/family compared to respective population; * and \# comparisons between specific countries; Number in respective boxes: Citations.

and cultural factors and the degree to which the microbiome is shaped by each of these factors remains debated. It is not clear yet which factor plays a dominant role in shaping the microbiomenature or nurture, host genetics or his environment, traditions and life-style? The present review has made an attempt toward identification of the factors responsible for geography-based alterations in microbial communities. Possible links between the microbiome structure and the disease susceptibility of the host population has also been discussed. Our work clearly indicates the need for the global association studies between human microbiota and different geographic locations for a proper assessment of the relative importance of diet, ancestry and locations in sculpting the human microbiome architecture.

\section{GASTROINTESTINAL MICROBIOME}

In humans, the gastrointestinal microbiome, especially the gut microbiota (GM), has the largest number of microbes and the greatest variety of species compared to other body habitats.
The GM constituents, as predicted from the fecal microbiome, vary substantially across healthy individuals (Consortium, 2012). GM dysbiosis is associated with numerous metabolic and inflammatory disorders like obesity, diabetes, allergy etc. (Adlerberth and Wold, 2009; Armougom et al., 2009; Anderson et al., 2011; Clemente et al., 2012). Cross-population studies on GM usually attempt to address any of the two major issues: (a) influence of host ethnicity and/or life-style on microbiome structure (De Filippo et al., 2010; Yatsunenko et al., 2012; Mardanov et al., 2013; Tyakht et al., 2013; Schnorr et al., 2014; Martinez et al., 2015; Morton et al., 2015; Obregon-Tito et al., 2015; Rampelli et al., 2015; Sankaranarayanan et al., 2015; Gomez et al., 2016) and (b) plausible correlations between variations in GM structure and disease susceptibility (O'Keefe et al., 2007; Ou et al., 2013).

Table 1 provides the salient features of some cross-population studies that highlighted the influence of host ancestry/life-style on fecal microbiome structure, and Figure 2 depicts the trends in evolution of GM with change in host life-style, as observed in these studies. In most of these studies, investigators compared 


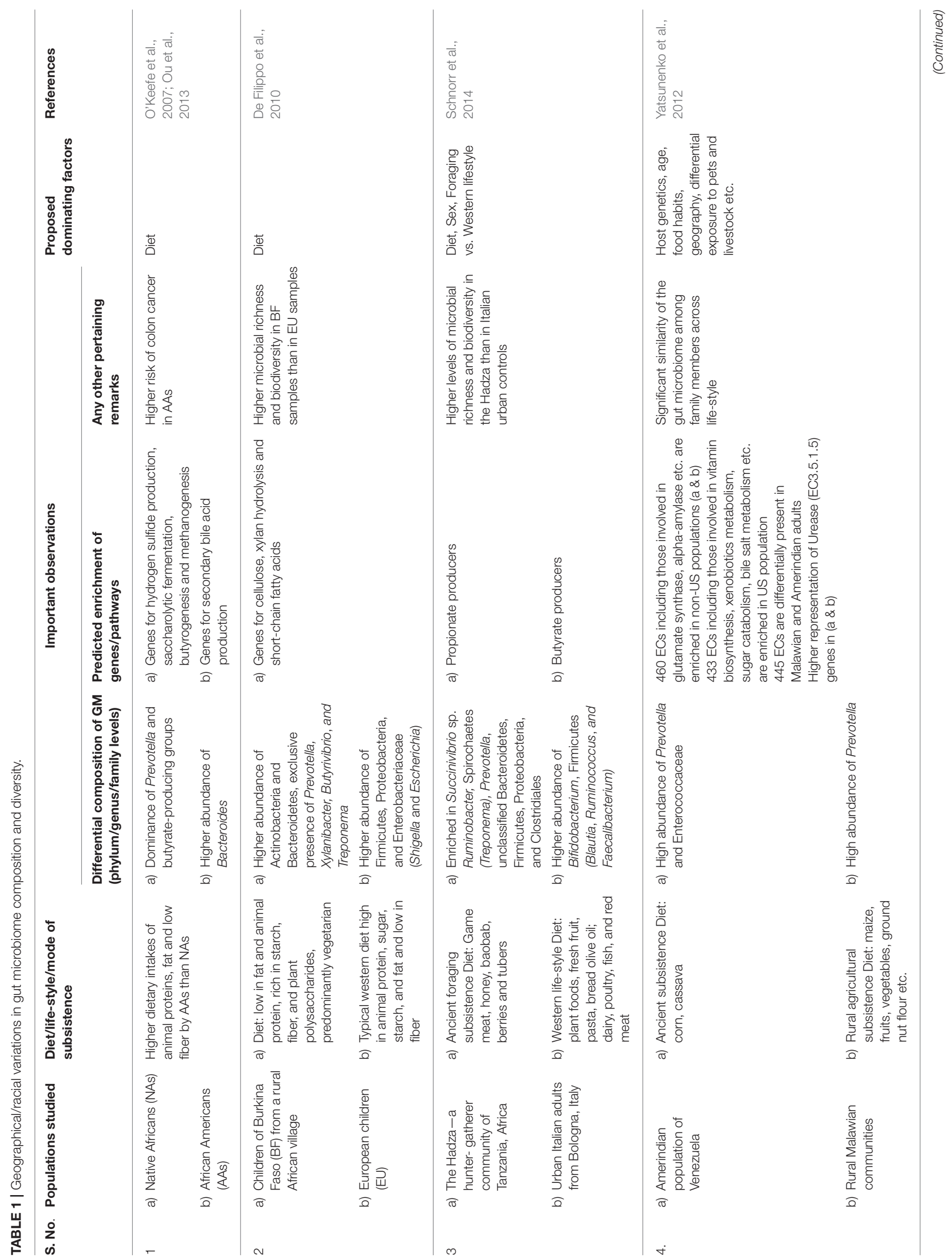




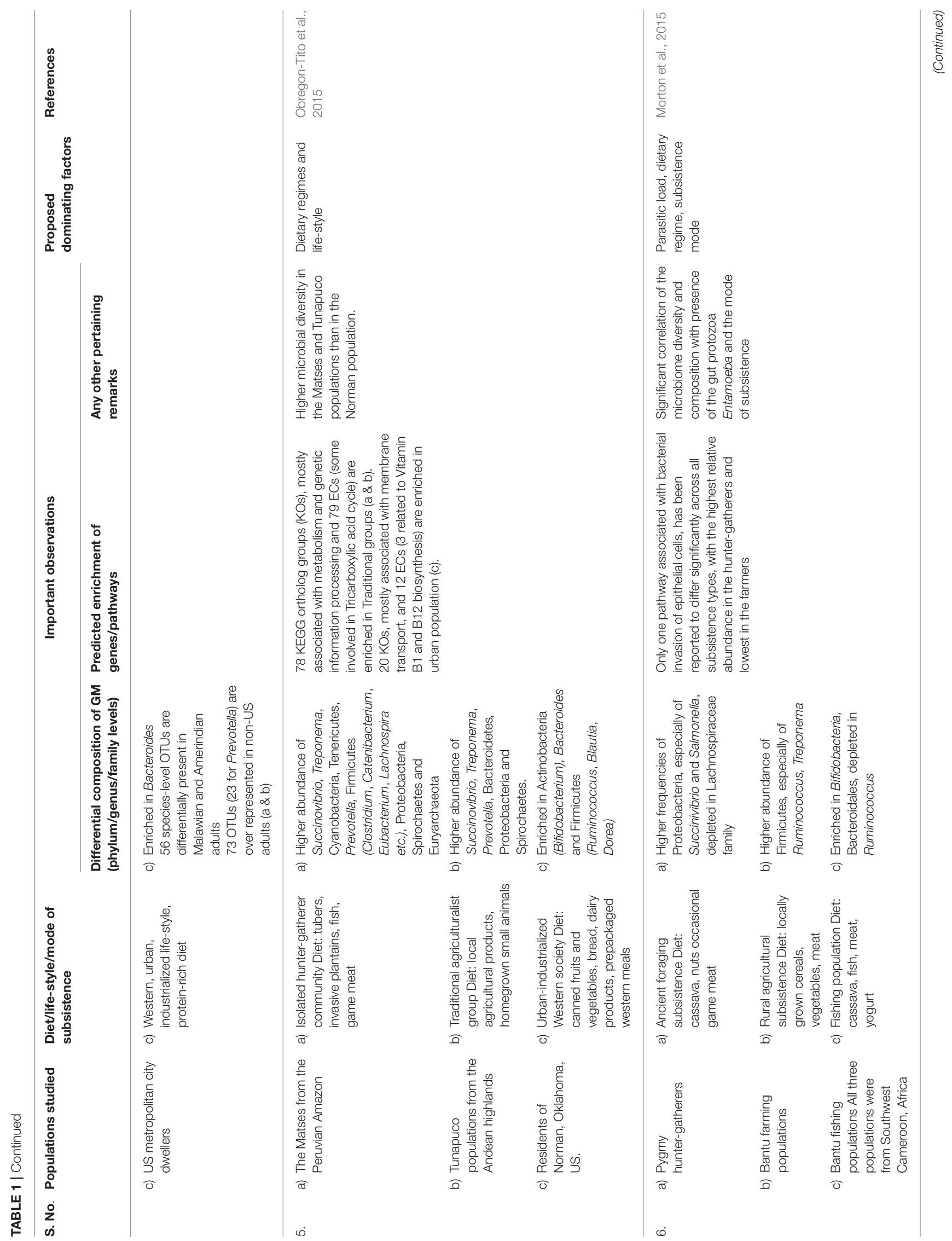


the GM diversity and composition in two or more representative communities from three distinct subsistence modes: (a) a remote hunter-gatherer population such as the Hadza from Tanzania, Pygmies from Central Africa, the Matses from Peru and Amerindians from Venezuela (O'Keefe et al., 2007; De Filippo et al., 2010; Grzeskowiak et al., 2012; Ou et al., 2013; Schnorr et al., 2014; Morton et al., 2015; Obregon-Tito et al., 2015); (b) a traditional farming or fishing population of localities like the Bantus of Africa, the Tunapuco populations of the Andean highlands or the rural Malawian communities (Gomez et al., 2016); and (c) a representative group of the western (US/European) urban industrialized society (De Filippo et al., 2010; Grzeskowiak et al., 2012; Mardanov et al., 2013; Tyakht et al., 2013; Greenhill et al., 2015; Sankaranarayanan et al., 2015). The hunter-gatherer communities primarily rely on starchy foods like tubers or cassava, plants, nuts, wild game, and honey for sustenance. These remote foragers are known to suffer from multiple infections of gastrointestinal pathogens/parasites, but have little or no access to modern healthcare or medical facilities (Morton et al., 2015; Gomez et al., 2016). The diet of the traditional agriculturalists, on the contrary, is similar to that of people of the Neolithic age, when people switched over from the nomadic lifestyle to settlement in villages, food-crop cultivation and domestication of animals (and fishing and trading at a later stage). People of US metropolitan cities or European countries are typical representatives of the WEIRD societies characterized by urban, industrialized life-style, refined high-protein high-fat diet, improved sanitation and hygiene practices and habitual use of antibiotics and other drugs (De Filippo et al., 2010; Grzeskowiak et al., 2012; Mardanov et al., 2013; Tyakht et al., 2013; Greenhill et al., 2015; Sankaranarayanan et al., 2015). In another study Bengtsson-Palme et al. showed that the gut microbiome varies in abundance of antibiotic resistance genes in their genomes across different geographical populations and acts as a transporter for these genes when people travel from one place to another (Bengtsson-Palme et al., 2015). Some of the cross-population microbiome studies suggested plausible correlations between GM composition and disease susceptibility (Table 1). Fecal metagenomic markers for T2D were found to differ between European and Chinese populations, suggesting that fecal metagenomic predictive tools for T2D should be specific for different geographical populations (Karlsson et al., 2013).

All these studies revealed some general trends in evolution of human GM with gradual transition in host subsistence pattern (Figure 2). The most apparent trend is significantly higher microbial diversity in guts of foraging people and its gradual reduction with westernization in the host community (Schnorr et al., 2014). For instance, the microbiome profiles of the agriculturalist Bantu population exhibit an intermediate state between the microbiomes of Pygmy hunter-gatherers and those of US individuals (Obregon-Tito et al., 2015; Gomez et al., 2016). It has been proposed that the high taxonomic diversity in the wandering foragers might have endowed their gastrointestinal ecosystem with greater stability and functional flexibility, enabling them to withstand the perpetual presence of pathogens or parasites and to respond to fluctuations in diet due 


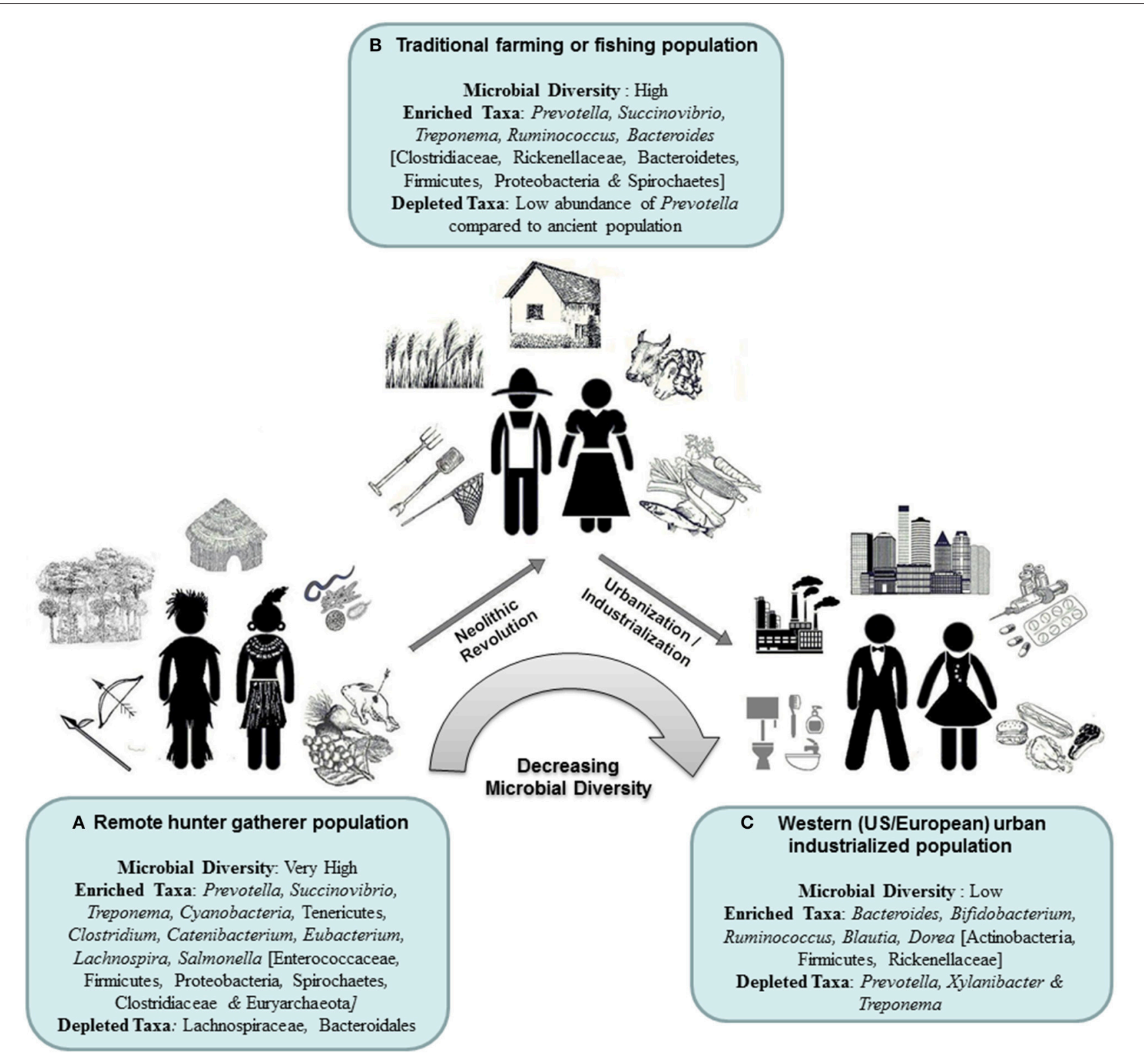

FIGURE 2 | Gradual transition of the gut microbiota composition with changes in the host subsistence strategies.

to unpredictable and seasonally dependent food supply (Morton et al., 2015).

Divergence in the GM composition, especially the PrevotellaBacteroides trade-off between the hunter-gatherers and western people is often attributed to their dietary specialization (O'Keefe et al., 2007; Ou et al., 2013; Gomez et al., 2016). Prevotella is thought to enhance the ability to digest and extract valuable nutrition from fibrous plant foods and Treponema also might be advantageous to nutritional acquisition of traditional people because of its fibrinolytic specializations (ObregonTito et al., 2015). As compared to foraging populations, the agriculturalists and urban industrialized people exhibit an appreciable enrichment in carbohydrate- and xenobioticprocessing presumably due to their access to more digestible sugars and therapeutic drugs (Gomez et al., 2016).

The role of host genetics in shaping the GM is not very clear yet (Goodrich et al., 2016), though there were some reports on ethnicity or geography-specific variations in GM (Yatsunenko et al., 2012). Yatsunenko et al. noticed a basal level of influence of family and shared environment on microbiome structure, which was found to be fairly consistent across US, Malawian and Amerindian communities (Yatsunenko et al., 2012). Analysis of fecal microbiota of infants, genetically at high risk for Type I Diabetes at six clinical centers in Europe 
(Finland, Sweden, and Germany) and the U.S. (Colorado, Washington state, and Georgia/Florida) revealed significant association of geographical origin with the diversity and the relative abundance of different bacterial genera, especially of Bifidobacterium, Veillonella, Faecalibacterium, Streptococcus, and Akkermansia in GM (Kemppainen et al., 2015). The study found significantly lower diversity in the fecal microbiota of Finland and Colorado babies, which was in good agreement with an earlier report on reduction in GM diversity in infants from northern European countries as compared to southern or central European infants (e.g., Sweden vs. Spain and Finland vs. Germany), irrespective of the delivery mode, breast-feeding and antibiotics exposure (Fallani et al., 2010). Geographical and population-specific factors might also have influenced IBD associated changes in gut ecosystems, as indicated by increase in abundance of Firmicutes in GM of IBD patients in European (Germany and Lithuania) population but not in South Asian (Indian) population (Rehman et al., 2016).

However, in many cases, the mode of subsistence exhibited specific microbiome signatures that seemed to overrule ethnic background. For instance, the Bantu-farming and Bantu-fishing communities though sharing the same genetic ancestry, show larger differences in gut microbiome profiles between themselves than between the Pygmies and Bantu-farming population (Morton et al., 2015). In the principal component analysis of genus-level taxa abundance profiles of GM, a clear separation was observed between three subsistence groups-the huntergatherer populations (Hadza and Matses from Africa and South America) the rural agriculturalists (Tunapuco, Malawi, and Venezuela from Africa and South America) and the urban industrial populations (from Europe and North America), which clearly indicated that the influence of diet/subsistence on GM may overrule the host ancestry and geographic origin (ObregonTito et al., 2015). Dominance of diet and other life-style factors over genetics/ethnicity is also apparent from the compositional differences in gut microbiome between Hadza men and womensignificantly higher abundance of Treponema in women and increased Eubacterium and Blautia in men-which may be attributed to the sexual division of labor and sex differences in dietary regimes (Schnorr et al., 2014). The effect of diet on gut microbiome is also apparent from the observation that porphyranase and agarase genes are specific to Japanese gut microbiome and absent from that of western population. It was proposed that these genes might have been acquired by gut bacteria of Japanese from marine bacteria through seaweed diet (Hehemann et al., 2010). A similarity in the gut microbiome composition (dominance of Bacteroidetes and Firmicutes) was observed between humans and domestic cattle and chimpanzees living within the same geographical location (Ellis et al., 2013).

The parasite burden in gut could also be a governing factor for gut ecosystem diversity in the Pygmy hunter-gatherers, Bantu farming and Bantu fishing populations, suggesting potential important interactions between the host immune system, gut microbiome, and gut parasites (Morton et al., 2015). Abundance of some predicted pathways derived from gut microbiome of entamoeba positive and negative individuals were found to be significantly $(P<0.05)$ different, for instance, pathways involved in biosynthesis of antibiotic tetracycline and yeast MAPK signaling pathways were found in higher abundance in gut microbiome of the BaAka pygmies, who often suffer from multiple infections of pathogenic gastrointestinal parasites (entamoeba) and gut microbiome of entamoeba negative individuals have the higher representation of cellular antigens pathways compared to entamoeba positive individuals. These observations advocate for the role of parasitism in shaping the gut microbiota (Morton et al., 2015).

There are many other studies demonstrating geography or ethnicity-specific divergences in GM composition (Figure 1). For instance, American community, both Japanese and Korean communities and Chinese community showed high abundances of Firmicutes, Actinobacteria, and Bacteroidetes, respectively in their GM (Nam et al., 2011). At the genus levels, Japanese (Nishijima et al., 2016) showed high abundances of Bifidobacterium and Clostridium, Chinese of Bacteroides and Korean of Prevotella and Faecalibacterium in their GM. Nishijima et al., showed dominance of Prevotella in Malawi, Venezuela, and Peru; Bacteroides in USA, China, Denmark, Spain, and France; Eubacterium in Russia; Clostridium in Sweden and Blautia in Austria (Nam et al., 2011; Nishijima et al., 2016). Bacteroides genus dominated in American and Jamaican populations while Prevotella genus dominated in Indian population (Kao et al., 2016). Ruminococcus, Roseburia, Veillonellaceae dominated in gut microbiome of healthy individuals from the Netherlands (Bonder et al., 2016). Three robust clusters (enterotypes) based on gut microbiome composition were identified by Arumugam et al. but populations specific variations were not well explored (Arumugam et al., 2011).

\section{ORAL MICROBIOME}

Microbes that colonize at several niches within our oral cavity after birth and form a stable ecosystem are collectively called oral microbiome. Diseases like periodontitis, teeth reduction, caries and even cancer are known to be associated with imbalance in oral microbiome composition. The fact that susceptibility to these diseases often shows ethnic biases, has inspired the investigators to explore the geographical variations in oral microbiome and its potential impact in oral health. Blekhman et al. observed a link between variation of microbiome composition in oral cavity and host genetic variation when different populations (African vs. American, African vs. European, African vs. Asian, American vs. European, and American vs. Asian) were compared by considering the $\mathrm{F}_{\mathrm{ST}}$ (Fixation Index) values. Similarly, Li et al. (2014) also showed that saliva microbiome of genetically different populations from Alaska, Germany and Africa were significantly different in alpha (within sample) and beta (between samples) diversity for microbiome composition in saliva. Mason et al. compared the oral microbiome composition between the healthy populations from major races/ethnic groups residing in the United States namely non-Hispanic blacks, non-Hispanic whites, Chinese and Latinos and found that 33 of 77 genera significantly differ 
in abundance between these four populations (Mason et al., 2013). The study demonstrated ethnicity-specific clustering of microbial communities in saliva and subgingival biofilms and using a machine-learning classifier, it had been possible to identify an individual's ethnicity from sub-gingival microbial signatures with a $100 \%$ sensitivity and $74 \%$ specificity in African American, 67 and 80\% in Latinos and 50 and 91\% in Caucasians (Mason et al., 2013). As African Americans and Caucasians share similar environmental factors including food, nutrition, and lifestyle over several generations, it was suggested that the distinct composition of their oral microbiome could be due to the variations in tooth and root morphologies across different ethnic populations as well as to their innate immune responses to infectious agents (Lavelle, 1970, 1971; Cruz et al., 2009; Dewhirst et al., 2010; Mason et al., 2013; Wade, 2013).

These observations contradicted an earlier study by Nasidze et al., who analyzed 16S rRNA sequences from saliva samples from 120 healthy individuals (10 individuals from each of 12 worldwide geographic locations), but did not find any definite correlation of the compositional (presence/absence) variation in saliva microbiota with geographical distances between locations, though abundances of certain genera were significantly different in specific locations (Nasidze et al., 2009). For instance, Enterobacter accounted for $28 \%$ of total composition in Congo population but was completely absent in samples from California, China, Germany, Poland, and Turkey. California and the Congo showed the largest differences between individuals, while Georgia and Turkey showed the smallest differences between individuals (Nasidze et al., 2009).

A comparative study on salivary microbiome composition in Alaskans, Germans, and Africans revealed more similarities between native Alaskans and Germans than between either group and Africans both at the genus and OTU levels ( $\mathrm{Li}$ et al., 2014). Abundance of the Firmicutes was highest in Alaskans and Germans, while in Africans, Proteobacteria was the most abundant phylum. Six common genera-Neisseria, Campylobacter, Granulicatella, Megasphaera, Selenomonas, and Actinomyces-were shared by both Alaskans and Africans but only three genera namely Actinobacillus, Aggregatibacter, and Capnocytophaga were shared by Germans and Africans (Li et al., 2014). Beta diversity was highest in Africans but alpha diversity in Germans (Li et al., 2014). Four Alaskan groups, located in different regions of Alaska and habituated to distinct diets, did not reveal any significant differences in their saliva microbiome composition. But substantial differences could be observed in the microbiome diversity among three African groups (Nasidze et al., 2011; Li et al., 2014). The Batwa Pygmies, a former huntergatherer group from Uganda, showed higher diversity in saliva microbiome than that in the agricultural groups from Sierra Leone and the Democratic Republic of Congo, which may be attributed to the ancient subsistence pattern and protein-rich diet of the pygmies (Nasidze et al., 2011; Li et al., 2014). People of Sierra Leone and Congo, who are geographically distant but have similar life style and diet, showed a higher degree of similarity to each other than with the Batwa. The Batwa population had low incidence of dental caries, presumably due to higher occurrences of Haemophilus, which is known to play an important role in
$\mathrm{pH}$ homeostasis in oral cavity (Nasidze et al., 2011; Li et al., 2014).

\section{RESPIRATORY TRACT MICROBIOME}

The human respiratory tract is subdivided physiologically into two parts i.e., Upper Respiratory Tract (URT) composed of oropharynx, nasopharynx and nasal cavity and Lower Respiratory Tract (LRT) containing lungs. LRT were traditionally assumed to be sterile, when identification of microorganisms relied on culture based methodologies. But recent advancement of culture independent molecular methodology changed this notion and indicated the presence of microbes in LRT (Charlson et al., 2011). Discovery of human airways microbiome opened the opportunity for understanding the disease onset, exacerbation and progression of chronic respiratory diseases which might be associated with dysbiosis in microbiome (Martin et al., 2015).

Most studies of airways microbiome characterization were focused on URT and few studies of LRT microbiome characterization in healthy human have been restricted to Western European and North American populations (Charlson et al., 2011). The healthy lung microbiome was found indistinguishable from URT except the exclusive presence of Tropheryma whipplei in lungs microbiome (Charlson et al., 2011). URT microbiome has been characterized in geographically diverse populations from USA, South Korea (Yi et al., 2014), Netherlands (de Steenhuijsen Piters et al., 2016), and Canada (Stearns et al., 2015) and found mostly similar in all populations. URT microbiota is usually dominated by Firmicutes, Actinobacteria, Proteobacteria, Bacteroidetes, and Fusobacteria phyla, which contain species from Streptococcus, Neisseria, Gemella, Corynebacterium, Alloiococcus, and Haemophilus genera. The healthy adult lung (LRT) microbiome in US population was dominated by Bacteroidetes, Firmicutes and Proteobacteria phyla, which included the species from Streptococcus, Veillonella, Prevotella, Pseudomonas, Haemophilus, and Neisseria (Charlson et al., 2011; Morris et al., 2013; Allen et al., 2014; Botero et al., 2014; Bassis et al., 2015; Dickson et al., 2015a,b; Tarabichi et al., 2015). There is no significant difference identified in lung microbiome from eight geographically different cities in USA (Morris et al., 2013).

\section{SKIN MICROBIOME}

The skin is the largest body organ of the human, composed of distinct habitats that differ by skin thickness, folds, the density of hair follicles and type of glands. Millions of microbes, mostly commensal, colonize on skin and disruption of this healthy microbiota may cause various diseases (Noble, 1984; Roth and James, 1989; Chiller et al., 2001; Fredricks, 2001; Cogen et al., 2008; Tagami, 2008; Grice and Segre, 2011). Studies suggested that diseases like atopic dermatitis, psoriasis, rosacea, acne etc. are often caused not because of pathogens but due to disruption in normal skin microbiota (Ong et al., 2002; Nomura et al., 2003a,b; de Jongh et al., 2005; Gudjonsson et al., 2009). 
Microbial colonization on skin depends on various factors like age, anatomical location, gender, climate, geographical location and exogenous environmental factors (Chen and Tsao, 2013). Understanding of variation in skin microbiome composition in different ethnic or geographical population may explore the reason of variation in susceptibility to certain pathologies or skin disorders in diverse populations. Studies on hand microbiota of women from US and Tanzania showed higher abundances of Propionibacteriaceae, Staphylococcaceae and Streptococceacae families in US population, and that of soilassociated Rhodobacteraceae and Nocardioidaceae in Tanzanian women (Hospodsky et al., 2014). This geographical diversity in skin microbiome might be ascribed to distinct environment and life style in both countries (Hospodsky et al., 2014). US population spends majority of time indoors in contact with dry surfaces, while Tanzanian population performs daily activities in open air in contact with soil, water etc. (Hospodsky et al., 2014).

Comparison of forearm skin specimens from healthy Amerindians in the Venezuelan Amazon and healthy persons in New York and Colorado, US, showed a significant difference in microbiota composition between these two communities (Blaser et al., 2013). The US samples were dominated by Propionibacterium. Amerindians were clustered into two groups. One showed bacterial diversity similar to the US community, though it was dominated by Staphylococcus. The other group contained a broad range of Proteobacteria with substantially more diversity than the US population and the first group of Amerindians (Blaser et al., 2013). The Amerindians selected for this study represented a population in transition that shifted two or three generations ago from a nomadic hunter-gatherer life-style to permanent homes with access to certain aspects of modern life, yet with a relatively traditional diet (Blaser et al., 2013). However, the factors that distinguished two groups of Amerindian cutaneotypes could not be identified. No definite association of two groups could be found with age, gender, body mass index, relation to drinking water, bathing, use of soap, or other factors (Blaser et al., 2013).

A study on Chinese population showed the considerable variations in skin microbiota between urban and rural population (Ying et al., 2015). Trabulsiella was more abundant in urban population than rural, especially on sites including volar forearm, glabella and back of hands (Ying et al., 2015). Propionibacterium showed variations in abundance based on skin site and gender between urban and rural dwellers (Ying et al., 2015). In women, Propionibacterium on glabella showed the higher abundance in urban than rural population, while Corynebacterium exhibited the reverse trend (Ying et al., 2015). The rural adults and elderly people that participated in the study were all agricultural fieldworkers and hence were exposed to soil, aquatic and other environmental microbial sources that could alter their skin microbiome composition (Ying et al., 2015). On the other hand, most urban subjects had indoor occupations and thus their skin microbiome are predominantly human-derived with little contribution from environmental sources (Ying et al., 2015).

A study on Hong Kong population has implemented the concept of pan microbiome-the total number of microbial species in a specific population (Leung et al., 2015). In this study, the investigators found a steady increase in the size of the pan microbiome, as populations from US, Tanzania and China were included in the dataset, indicating variations in skin microbiome across the countries (Leung et al., 2015).

\section{VAGINAL MICROBIOME}

The urogenital tract (UGT) microbiome has been characterized mainly in samples from female subjects derived from vaginal sites. Lactobacillus species are known to be the major component of the healthy vaginal microbiota but some studies indicated that Gardnerella, Atopobium, Prevotella, Pseudomonas, or Streptococcus species are predominant in some healthy women instead of Lactobacillus (Hyman et al., 2005; Zhou et al., 2007; Fettweis et al., 2014).

Vaginal microbiome of North American-Asian, North American-white and North American-black women was dominated by Lactobacillus (51-96\%) but in Belgium population vaginal microbiome was dominated by Bacteroides (34\%) (Ravel et al., 2011; Verstraelen et al., 2016).

In 2007, a study by Zhou et al. demonstrated significant divergences in vaginal microbiome composition between healthy Caucasian and black women of reproductive age in North America. Microbial communities dominated by Lactobacillus species were found to be common in Caucasian women, while the communities dominated by Atopobium and a diverse array of phylotypes from the order Clostridiales prevailed in black women (Zhou et al., 2007). A 16S rRNA gene survey of vaginal samples of 396 asymptomatic North American women from four ethnic groups (white, black, Hispanic, and Asian) elucidated existence of five distinct groups of vaginal microbiome profiles: four were dominated by Lactobacillus crispatus, $L$. gasseri, L. iners, or L. jensenii, while the fifth contained lower proportions of lactic acid bacteria and higher proportions of strictly anaerobic organisms (Ravel et al., 2011). The proportions of each community group varied considerably among the four ethnic groups, with Lactobacillus prevailing among the Asian and white women and anaerobic species being abundant in black and Hispanic women (Ravel et al., 2011). A similar type of study on apparently healthy Japanese women in Tokyo, and White and Black women from North America showed incidences of vaginal communities with several non-Lactobacillus species gradually increase from White to Japanese to Black populations (Zhou et al., 2010).

Analysis of vaginal microbiome composition in healthy and diseased African-American and European-American women by Fettweis et al. reconfirmed that European-American vaginal microbiome have the low bacterial diversity dominated by Lactobacillus species, while vaginal microbiome of AfricanAmerican women is more diverse in nature and dominated by Gardnerella vaginalis and the uncultivated bacterial vaginosisassociated bacterium-1 (BVAB1) (Fettweis et al., 2014). These observations comply with higher occurrences of bacterial vaginosis among African-American women than among European-American population. Moreover, the prevalence of various bacterial taxa that are known to be associated with 
microbial invasion of the amniotic cavity and preterm birth such as Mycoplasma, Gardnerella, Prevotella, Sneathia etc. also differed between the two ethnic groups (Fettweis et al., 2014). Almost similar type of divergences in vaginal microbiome has also been observed between Belgian (Verstraelen et al., 2016) and Canadian women (Albert et al., 2015), with L. crispatus. L. iners, and Prevotella prevailing in Belgian population and $L$. iners, $L$. jensenii, and $G$. vaginalis in Canadian population.

Whether the variation along ethnic lines is a reflection of genetics or environment remains a matter of conjecture. Zhou et al. proposed that host genetic factors, including the innate and adaptive immune systems, may be more important in shaping the composition of vaginal microbiota than the cultural and behavioral differences among ethnic groups such as multiple sex partners, douching, and the use of contraception devices (Zhou et al., 2007). The vaginal bacterial communities of Japanese women resemble those of women in other racial groups (white and black women from North America) (Zhou et al., 2010). But in other ethnic populations (African American and European) Fettweis et al. found significant correlation not only of ethnicity, but also of no-pregnancy and less-alcohol use with the higher relative abundance of bacterial vaginosis associated species (Fettweis et al., 2014).

\section{GEOGRAPHICALLY CONSERVED CORE MICROBIOME}

Apart from a detail discussion of geography, dietary habits, ethnicity or local environment (rural/urban) specific variations in human microbiome, the present article also aims to characterize the geographical-core (present in all populations under the study) microbiota at different body habitats of human. This is totally based on the published data on relative abundance of microbial communities at distinct body niches in healthy human subjects (Figure 3, Tables S1-S3). The core microbiota of a specific body site of human refers to the set of the genera, which are commonly found in that specific body site of all populations studied so far, irrespective of their geographic locations, ethnic background or places of dwelling. In 2016, Falony et al. also identified a core microbiome (based on individuals) but in this present analysis we considered the populations instead of individuals for estimating the geographical-core microbiome (Figure 3, Tables S1-S3) (Falony et al., 2016). Size of the geographical core indicates the effect of geographical factors on microbiome composition that might be useful to understand the geographical exclusiveness of a specific microbial community.

In case of stool derived microbiome, 25 genera are found to be common in all populations from 12 different countries, though the abundances of these genera have been found to vary substantially across populations (Figure 3A, Table S1) (Qin et al., 2010, 2012; Yatsunenko et al., 2012; Karlsson et al., 2013; Tyakht et al., 2013; Zeller et al., 2014; Feng et al., 2015; Obregon-Tito et al., 2015; Nishijima et al., 2016). For instance, Bacteroides are dominant in people from USA (38\%), China (39\%), Spain (23\%), and Denmark (20\%) compared to Malawi (3\%), Peru (1\%), Russia (8\%), Venezuela (3\%), and Sweden (8\%), while Prevotella is enriched in Malawi (32\%), Peru (14\%), and Venezuela (24\%) as compared to USA (4\%) and Canada (4\%) (Qin et al., 2010, 2012; Yatsunenko et al., 2012; Karlsson et al., 2013; Tyakht et al., 2013; Zeller et al., 2014; Feng et al., 2015; Obregon-Tito et al., 2015; Nishijima et al., 2016).

Salivary microbiome as a part of the oral microbiome are characterized in 15 populations from 13 geographically diverse countries, but only 15 genera are commonly present in all 15 populations (Nasidze et al., 2009, 2011; Li et al., 2014). In Congo, Sierra Leone, Uganda and Bolivia population, core microbiota covers only $41,33,58$, and $65 \%$ of total salivary microbiota, respectively (Figure 3B, Table S2). This variation in relative abundances of core microbial communities indicate the effect of population specific factors like diet, genetics, life style, use of antibiotics, occupation behaviors etc.

Different number of genera has been found in vaginal microbiome of various individuals from 7 different populations from 4 countries (Belgian, US-Asian, US-white, US-Hispanic, US-black, Canadian, and Japanese). Out of all characterized genera only 5 genera $(>0.1 \%)$ are shared among all seven populations (Figure 3C, Table S3) (Zhou et al., 2010; Ravel et al., 2011; Albert et al., 2015; Verstraelen et al., 2016). All populations except the Belgians $(\sim 16 \%)$ are dominated $(>60 \%)$ by Lactobacillus. Four ethnic populations from USA were clustered into two groups (White/Asian and Black/Hispanic) in their vaginal microbiome composition (Figure 3C).

With a view to identify the primary causes of variations in the core microbiome composition (relative abundances) of specific body niches across different populations, we have conducted the Principal Component Analysis (PCA) of the core microbiota composition in stool, saliva and vagina using abundances of core microbiome of 12, 15, and 7 different populations, respectively. Figure 3 depicts the Axis 1 vs. Axis 2 plot of PCA of the stool (Figure 3A), saliva (Figure 3B) and vaginal (Figure 3C) microbiome of different populations, where the directions of increase of various bacterial genera are indicated by specific arrows, which indicates the population specific dominance of a specific microbial community (Figure 3). These observations clearly indicate that though there exists a core microbiome in each specific body-niche, distinct bacterial genus/genera dominate in healthy individuals of different geographical and/or ethnic populations. Figure 3 showed that most of the variance is accounted by first two principal components in vaginal microbiome $(\sim 99 \%)$, stool $(\sim 85 \%)$, and saliva $(\sim 75 \%)$. All geographical populations are grouped in different clusters on respective PCA plots for example, gut microbiome is grouped in 3 main clusters as shown in Figure 3A (Cluster 1 [Bacteroides dominance]: Denmark, Spain, USA and China; Cluster 2 [Prevotella dominance]: Peru, Venezuela, Russia and Malawi; Cluster 3 [Ruminococcus and Blautia dominance]: Austria, Sweden, Japan, and France); saliva microbiome also clustered into 3 groups as shown in Figure 3B which comprise differential dominance of microbial genera for example, Cluster 1: Streptococcus, Neisseria, and Haemophilus (USA-Louisiana, USA-California, Germany, and China); Cluster 2: Prevotella and Veillonella (USA-Alaska, South Africa, Poland, Turkey, Philippines, and Argentina); the vaginal microbiome is clustered 

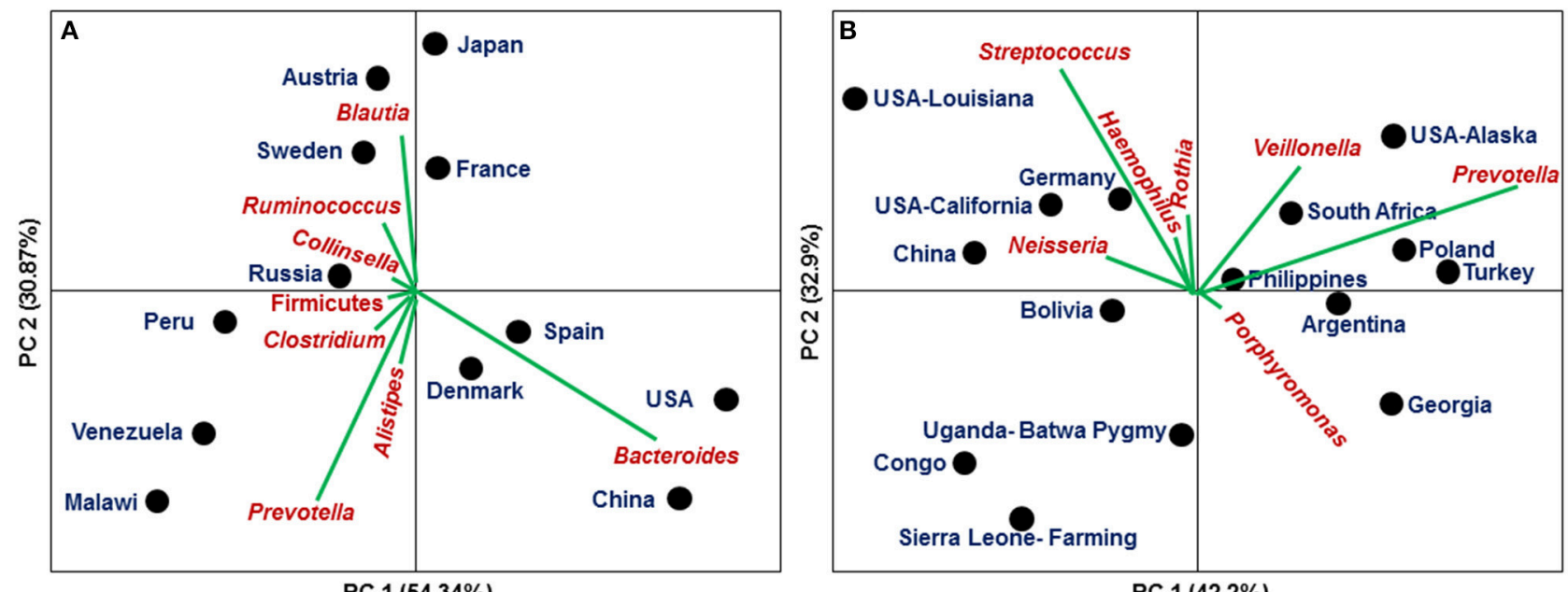

PC $1(54.34 \%)$

PC $1(42.2 \%)$

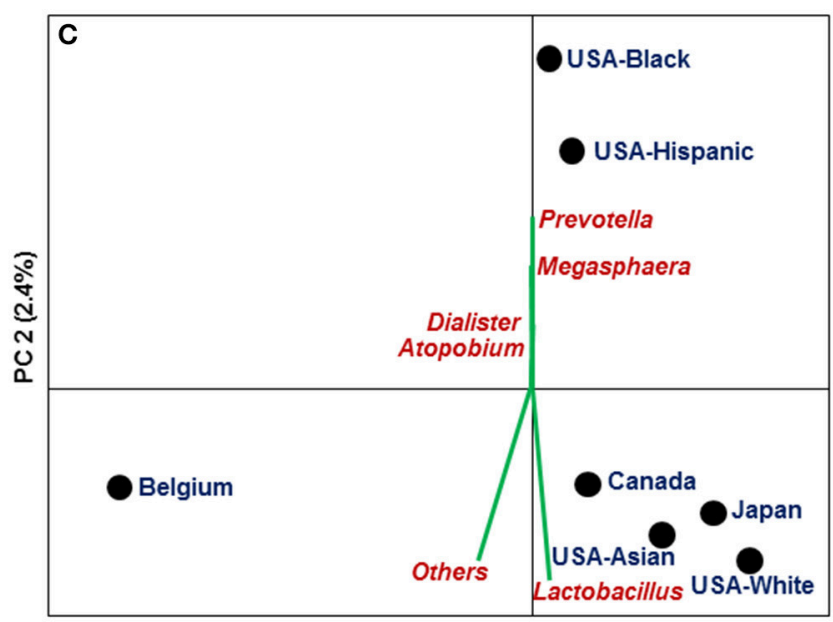

PC $1(97 \%)$

FIGURE 3 | Principal Component Analysis based on relative abundances of core microbiota among different countries/populations derived from a specific body niche (population wise data shown in Tables S1-S3): (A) Gut (B) Oral cavity (C) Vagina.

into 2 groups (Cluster 1 [Lactobacillus dominance]: USA-Black and USA-Hispanic; Cluster 2 [Prevotella dominance]: Canada, Japan, USA-Asian and USA-White) but Belgium population is segregated from both groups (Figure 3C).

\section{SUMMARY}

Studies conducted on human microbiome so far revealed some general trends observed in variations in micro-ecology at distinct body habitats across various countries/populations around the world (Figure 1). It appears that there had been a gradual transition in the gross compositional structure and decrease in diversity of the human microbiome, especially in the gut microbiota, as the human populations passed through three stages of subsistence-foraging, rural farming and industrialized urban life. Higher microbial diversity in nomadic hunter-gatherers had probably endowed them with greater stability and flexibility, enabling them to cope with challenging ecology. Changes in human diet, lifestyle and traditions brought about by the Neolithic revolution have been reflected in the microbiota, especially in the gut microbiome of the traditional agriculturalists. With time, urbanization and industrialization have gradually led to modern people of developed countries, adapted to indoor-based secured life-style, consumption of refined high protein foods, improved sanitation, less exposure to soil, forest or domestic animals and habitual use of antibioticsall having a dramatic impact on the functional role of the western microbiome.

Much of the mutualistic functions of the ancestral human microbiome that could have facilitated our primitive forerunners to fight and survive against adverse environment are no more required and a substantial part of the ancient microbial ensemble that our ancestors shared has probably been lost through the adoption of modern urban, industrial, western lifestyle. For instance, exclusive presence of Treponema in the gut of all 
traditional people studied to date (and also in non-human primates), but not in Western urban populations suggested that Treponema might be one of the symbionts lost in present-day urban-industrialized societies. This microbial simplification has probably left us with depleted microbiome, deficient in beneficial microbes that once might have played important metabolic roles in human health and this, perhaps, explains the rise of several "diseases of civilization" like allergies, diabetes, asthma, obesity, inflammatory bowel disease and so on.

The debate on "nature" vs. "nurture" remains to be resolved. Some of the geographical/racial variations in microbiome structure have been attributed to differences in host genetics and innate/adaptive immunity, while in many other cases, cultural/behavioral features like diet, hygiene, environmental exposure etc. overshadow genetics. Especially the diet and subsistence mode of the host population often inscribe their signatures in the gut microbiome diversity and composition, irrespective of the geographic origin, ethnicity or local environment of host population. The fact that despite large geographic and ethnic divergences, the hunter-gatherer populations like the Hadza from Africa and the Matses from South America or the rural agriculturalists like the Tunapuco from Africa and the Malawi and Venezuela from South America exhibit similarity in their gut microbiome structure advocates for the dominance of nurture over nature (Nam et al., 2011; Schnorr et al., 2014). On the contrary, host genetics and immunity are considered to be the major factors in shaping the UGT (vaginal) bacterial profiles, though hygiene, mode of pregnancy or alcohol addiction seems to have substantial influences (Zhou et al., 2010). Taken together, these conjectures motivate the need for larger association studies to assess the relative importance of host ancestry, diet, hygiene and other life-style factors in sculpting the human microbiome architecture.

\section{CONCLUSION}

Reports on ethnicity or population-specific variations in human microbiome composition question the universality of the microbiome-based therapeutic strategies and recommend

\section{REFERENCES}

Adlerberth, I., and Wold, A. E. (2009). Establishment of the gut microbiota in Western infants. Acta Paediatr. 98, 229-238. doi: 10.1111/j.1651-2227.2008.01060.x

Albert, A. Y., Chaban, B., Wagner, E. C., Schellenberg, J. J., Links, M. G., van Schalkwyk, J., et al. (2015). A study of the vaginal microbiome in healthy canadian women utilizing cpn60-based molecular profiling reveals distinct gardnerella subgroup community state types. PLoS ONE 10:e135620. doi: 10.1371/journal.pone.0135620

Allen, E. K., Koeppel, A. F., Hendley, J. O., Turner, S. D., Winther, B., and Sale, M. M. (2014). Characterization of the nasopharyngeal microbiota in health and during rhinovirus challenge. Microbiome 2:22. doi: 10.1186/2049-2618-2-22

Anderson, C. A., Boucher, G., Lees, C. W., Franke, A., D’Amato, M., Taylor, K. D., et al. (2011). Meta-analysis identifies 29 additional ulcerative colitis risk loci, increasing the number of confirmed associations to 47. Nat. Genet. 43, 246-252. doi: $10.1038 / \mathrm{ng} .764$ for geographically tailored community-scale approaches to microbiome engineering. Generic microbiome manipulations, designed on the basis of studies on WEIRD societies, may have unintended, and even adverse consequences in non-western populations. However, designing a geographically tailored therapeutic approach would need an in-depth understanding of how population and environmental parameters can affect the microbial communities and their metabolic potentials, which, we hope, may be attained in near future through construction of pan microbiome of human populations around the globe.

\section{AUTHOR CONTRIBUTIONS}

VG developed the first draft of this review. SP provided suggestions for expansion and new direction and final editing. CD guided, coordinated the study and revised the manuscript critically for important intellectual content. All authors read and approved the final manuscript.

\section{FUNDING}

This work has been supported by the Council of Scientific and Industrial Research (CSIR), Government of India. (CSIR Network projects GENESIS (BSC0121) and HUM (BSC0119). SP is supported by Ramanujan Fellowship from Department of Science and Technology (DST), Govt. of India.

\section{ACKNOWLEDGMENTS}

We thankfully acknowledge the Narendrakumar M. Chaudhari for his contribution in preparation of figures in this MS.

\section{SUPPLEMENTARY MATERIAL}

The Supplementary Material for this article can be found online at: http://journal.frontiersin.org/article/10.3389/fmicb. 2017.01162/full\#supplementary-material 
Blaser, M. J., Dominguez-Bello, M. G., Contreras, M., Magris, M., Hidalgo, G., Estrada, I., et al. (2013). Distinct cutaneous bacterial assemblages in a sampling of South American Amerindians and US residents. ISME J. 7, 85-95. doi: 10.1038 /ismej.2012.81

Blekhman, R., Goodrich, J. K., Huang, K., Sun, Q., Bukowski, R., Bell, J. T., et al. (2015). Host genetic variation impacts microbiome composition across human body sites. Genome Biol. 16:191. doi: 10.1186/s13059-015-0759-1

Bonder, M. J., Tigchelaar, E. F., Cai, X., Trynka, G., Cenit, M. C., Hrdlickova, B., et al. (2016). The influence of a short-term gluten-free diet on the human gut microbiome. Genome Med. 8:45. doi: 10.1186/s13073-016-0295-y

Botero, L. E., Delgado-Serrano, L., Cepeda, M. L., Bustos, J. R., Anzola, J. M., Del Portillo, P., et al. (2014). Respiratory tract clinical sample selection for microbiota analysis in patients with pulmonary tuberculosis. Microbiome 2:29. doi: 10.1186/2049-2618-2-29

Brooks, J. P., Edwards, D. J., Harwich, M. D., Rivera, M. C. Jr., Fettweis, J. M., Serrano, M. G., et al. (2015). The truth about metagenomics. quantifying and counteracting bias in 16S rRNA studies. BMC Microbiol. 15:66. doi: 10.1186/s12866-015-0351-6

Castellarin, M., Warren, R. L., Freeman, J. D., Dreolini, L., Krzywinski, M., Strauss, J., et al. (2011). Fusobacterium nucleatum infection is prevalent in human colorectal carcinoma. Genome Res. 22, 299-306. doi: 10.1101/gr.126516.111

Charlson, E. S., Bittinger, K., Haas, A. R., Fitzgerald, A. S., Frank, I., Yadav, A., et al. (2011). Topographical continuity of bacterial populations in the healthy human respiratory tract. Am. J. Respir. Crit. Care Med. 184, 957-963. doi: 10.1164/rccm.201104-0655OC

Chen, Y. E., and Tsao, H. (2013). The skin microbiome: current perspectives and future challenges. J. Am. Acad. Dermatol. 69, 143-155. doi: 10.1016/j.jaad.2013.01.016

Chiller, K., Selkin, B. A., and Murakawa, G. J. (2001). Skin microflora and bacterial infections of the skin. J. Investig. Dermatol. Symp. Proc. 6, 170-174. doi: 10.1046/j.0022-202x.2001.00043.x

Clemente, J. C., Ursell, L. K., Parfrey, L. W., and Knight, R. (2012). The impact of the gut microbiota on human health: an integrative view. Cell 148, 1258-1270. doi: 10.1016/j.cell.2012.01.035

Cogen, A. L., Nizet, V., and Gallo, R. L. (2008). Skin microbiota: a source of disease or defence? Br. J. Dermatol. 158, 442-455. doi: $10.1111 /$ j.1365-2133.2008.08437.x

Consortium, T. H. M. P. (2012). Structure, function and diversity of the healthy human microbiome. Nature 486, 207-214. doi: 10.1038/nature11234

Cruz, G. D., Chen, Y., Salazar, C. R., and Le Geros, R. Z. (2009). The association of immigration and acculturation attributes with oral health among immigrants in New York City. Am. J. Public Health 99(Suppl. 2), S474-S480. doi: 10.2105/ajph.2008.149799

De Filippo, C., Cavalieri, D., Di Paola, M., Ramazzotti, M., Poullet, J. B., Massart, S., et al. (2010). Impact of diet in shaping gut microbiota revealed by a comparative study in children from Europe and rural Africa. Proc. Natl. Acad. Sci. U.S.A. 107, 14691-14696. doi: 10.1073/pnas.1005963107

de Jongh, G. J., Zeeuwen, P. L., Kucharekova, M., Pfundt, R., van der Valk, P. G., Blokx, W., et al. (2005). High expression levels of keratinocyte antimicrobial proteins in psoriasis compared with atopic dermatitis. J. Invest. Dermatol. 125, 1163-1173. doi: 10.1111/j.0022-202X.2005.23935.x

de Steenhuijsen Piters, W. A., Huijskens, E. G., Wyllie, A. L., Biesbroek, G., van den Bergh, M. R., Veenhoven, R. H., et al. (2016). Dysbiosis of upper respiratory tract microbiota in elderly pneumonia patients. ISME J. 10, 97-108. doi: 10.1038/ismej.2015.99

Dewhirst, F. E., Chen, T., Izard, J., Paster, B. J., Tanner, A. C., Yu, W. H., et al. (2010). The human oral microbiome. J. Bacteriol. 192, 5002-5017. doi: 10.1128/JB.00542-10

Dickson, R. P., Erb-Downward, J. R., Freeman, C. M., McCloskey, L., Beck, J. M., Huffnagle, G. B., et al. (2015a). Spatial variation in the healthy human lung microbiome and the adapted Island model of lung biogeography. Ann. Am. Thorac. Soc. 12, 821-830. doi: 10.1513/AnnalsATS.201501-029OC

Dickson, R. P., Erb-Downward, J. R., Martinez, F. J., and Huffnagle, G. B. (2015b). The microbiome and the respiratory tract. Annu. Rev. Physiol. 78, 481-504. doi: 10.1146/annurev-physiol-021115-105238

Ellis, R. J., Bruce, K. D., Jenkins, C., Stothard, J. R., Ajarova, L., Mugisha, L., et al. (2013). Comparison of the distal gut microbiota from people and animals in Africa. PLoS ONE 8:e54783. doi: 10.1371/journal.pone.0054783
Fallani, M., Young, D., Scott, J., Norin, E., Amarri, S., Adam, R., et al. (2010) Intestinal microbiota of 6-week-old infants across Europe: geographic influence beyond delivery mode, breast-feeding, and antibiotics. J. Pediatr. Gastroenterol. Nutr. 51, 77-84. doi: 10.1097/MPG.0b013e3181d1b11e

Falony, G., Joossens, M., Vieira-Silva, S., Wang, J., Darzi, Y., Faust, K., et al. (2016). Population-level analysis of gut microbiome variation. Science 352, 560-564. doi: $10.1126 /$ science.aad3503

Feng, Q., Liang, S., Jia, H., Stadlmayr, A., Tang, L., Lan, Z., et al. (2015). Gut microbiome development along the colorectal adenoma-carcinoma sequence. Nat. Commun. 6:6528. doi: 10.1038/ncomms7528

Fettweis, J. M., Brooks, J. P., Serrano, M. G., Sheth, N. U., Girerd, P. H., Edwards, D. J., et al. (2014). Differences in vaginal microbiome in African American women versus women of European ancestry. Microbiology 160, 2272-2282. doi: 10.1099/mic.0.081034-0

Fredricks, D. N. (2001). Microbial ecology of human skin in health and disease. J. Investig. Dermatol. Symp. Proc. 6, 167-169. doi: 10.1046/j.0022-202x.2001.00039.x

Gao, Z., Tseng, C. H., Strober, B. E., Pei, Z., and Blaser, M. J. (2008). Substantial alterations of the cutaneous bacterial biota in psoriatic lesions. PLoS ONE 3:e2719. doi: 10.1371/journal.pone.0002719

Garrett, W. S., Gallini, C. A., Yatsunenko, T., Michaud, M., DuBois, A., Delaney, M. L., et al. (2010). Enterobacteriaceae act in concert with the gut microbiota to induce spontaneous and maternally transmitted colitis. Cell Host Microbe 8 , 292-300. doi: 10.1016/j.chom.2010.08.004

Gerasimidis, K., Bertz, M., Quince, C., Brunner, K., Bruce, A., Combet, E., et al. (2016). The effect of DNA extraction methodology on gut microbiota research applications. BMC Res. Notes 9:365. doi: 10.1186/s13104-016-2171-7

Gomez, A., Petrzelkova, K. J., Burns, M. B., Yeoman, C. J., Amato, K. R., Vlckova, K., et al. (2016). Gut microbiome of coexisting BaAka pygmies and bantu reflects gradients of traditional subsistence patterns. Cell Rep. 14, 2142-2153. doi: 10.1016/j.celrep.2016.02.013

Goodrich, J. K., Davenport, E. R., Beaumont, M., Jackson, M. A., Knight, R., Ober, C., et al. (2016). Genetic determinants of the gut microbiome in UK twins. Cell Host Microbe 19, 731-743. doi: 10.1016/j.chom.2016.04.017

Greenhill, A. R., Tsuji, H., Ogata, K., Natsuhara, K., Morita, A., Soli, K., et al. (2015). Characterization of the gut microbiota of Papua New Guineans using reverse transcription quantitative PCR. PLoS ONE 10:e0117427. doi: 10.1371/journal.pone.0117427

Grice, E. A., and Segre, J. A. (2011). The skin microbiome. Nat. Rev. Microbiol. 9, 244-253. doi: 10.1038/nrmicro2537

Grzeskowiak, L., Collado, M. C., Mangani, C., Maleta, K., Laitinen, K., Ashorn, P., et al. (2012). Distinct gut microbiota in southeastern African and northern European infants. J. Pediatr. Gastroenterol. Nutr. 54, 812-816. doi: 10.1097/MPG.0b013e318249039c

Gudjonsson, J. E., Ding, J., Li, X., Nair, R. P., Tejasvi, T., Qin, Z. S., et al. (2009). Global gene expression analysis reveals evidence for decreased lipid biosynthesis and increased innate immunity in uninvolved psoriatic skin. J. Invest. Dermatol. 129, 2795-2804. doi: 10.1038/jid.2009.173

Hehemann, J. H., Correc, G., Barbeyron, T., Helbert, W., Czjzek, M., and Michel, G. (2010). Transfer of carbohydrate-active enzymes from marine bacteria to Japanese gut microbiota. Nature 464, 908-912. doi: 10.1038/nature 08937

Hospodsky, D., Pickering, A. J., Julian, T. R., Miller, D., Gorthala, S., Boehm, A. B., et al. (2014). Hand bacterial communities vary across two different human populations. Microbiology 160, 1144-1152. doi: 10.1099/mic.0.075390-0

Hyman, R. W., Fukushima, M., Diamond, L., Kumm, J., Giudice, L. C., and Davis, R. W. (2005). Microbes on the human vaginal epithelium. Proc. Natl. Acad. Sci. U.S.A. 102, 7952-7957. doi: 10.1073/pnas.0503236102

Islami, F., and Kamangar, F. (2008). Helicobacter pylori and esophageal cancer risk: a meta-analysis. Cancer Prev. Res. 1, 329-338. doi: 10.1158/1940-6207.CAPR-08-0109

Kao, C. C., Hsu, J. W., Dwarkanath, P., Karnes, J. M., Baker, T. M., Bohren, K. M., et al. (2016). Indian women of childbearing age do not metabolically conserve arginine as do American and Jamaican women. J. Nutr. 145, 884-892. doi: 10.3945/jn.114.208231

Karlsson, F. H., Tremaroli, V., Nookaew, I., Bergstrom, G., Behre, C. J., Fagerberg, B., et al. (2013). Gut metagenome in European women with normal, impaired and diabetic glucose control. Nature 498, 99-103. doi: 10.1038/nature12198 
Kemppainen, K. M., Ardissone, A. N., Davis-Richardson, A. G., Fagen, J. R., Gano, K. A., Leon-Novelo, L. G., et al. (2015). Early childhood gut microbiomes show strong geographic differences among subjects at high risk for type 1 diabetes. Diabetes Care 38, 329-332. doi: 10.2337/dc14-0850

Kostic, A. D., Gevers, D., Pedamallu, C. S., Michaud, M., Duke, F., Earl, A. M., et al. (2012). Genomic analysis identifies association of Fusobacterium with colorectal carcinoma. Genome Res. 22, 292-298. doi: 10.1101/gr.126573.111

Lavelle, C. L. (1970). Crowding and spacing within the human dental arch of different racial groups. Arch. Oral Biol. 15, 1101-1103. doi: 10.1016/0003-9969(70)90123-8

Lavelle, C. L. (1971). Mandibular molar tooth configurations in different racial groups. J. Dent. Res. 50:1353. doi: 10.1177/002203457105000 54501

Leung, M. H., Wilkins, D., and Lee, P. K. (2015). Insights into the pan-microbiome: skin microbial communities of Chinese individuals differ from other racial groups. Sci. Rep. 5:11845. doi: 10.1038/srep11845

Ley, R. E., Backhed, F., Turnbaugh, P., Lozupone, C. A., Knight, R. D., and Gordon, J. I. (2005). Obesity alters gut microbial ecology. Proc. Natl. Acad. Sci. U.S.A. 102, 11070-11075. doi: 10.1073/pnas.0504978102

Li, J., Quinque, D., Horz, H. P., Li, M., Rzhetskaya, M., Raff, J. A., et al. (2014). Comparative analysis of the human saliva microbiome from different climate zones: Alaska, Germany, and Africa. BMC Microbiol. 14:316. doi: 10.1186/s12866-014-0316-1

Mardanov, A. V., Babykin, M. M., Beletsky, A. V., Grigoriev, A. I., Zinchenko, V. V., Kadnikov, V. V., et al. (2013). Metagenomic analysis of the dynamic changes in the gut microbiome of the participants of the MARS-500 experiment, simulating long term space flight. Acta Nat. 5, 116-125.

Martin, C., Burgel, P. R., Lepage, P., Andrejak, C., de Blic, J., Bourdin, A., et al. (2015). Host-microbe interactions in distal airways: relevance to chronic airway diseases. Eur. Respir. Rev. 24, 78-91. doi: 10.1183/09059180.00011614

Martinez, I., Stegen, J. C., Maldonado-Gomez, M. X., Eren, A. M., Siba, P. M., Greenhill, A. R., et al. (2015). The gut microbiota of rural papua new guineans. composition, diversity patterns, and ecological processes. Cell Rep. 11, 527-538. doi: 10.1016/j.celrep.2015.03.049

Mason, M. R., Nagaraja, H. N., Camerlengo, T., Joshi, V., and Kumar, P. S. (2013). Deep sequencing identifies ethnicity-specific bacterial signatures in the oral microbiome. PLoS ONE 8:e77287. doi: 10.1371/journal.pone.0077287

Moossavi, S. (2014). The necessity for an Iranian gut microbiome initiative. Middle East J. Dig. Dis. 6, 109-110.

Morris, A., Beck, J. M., Schloss, P. D., Campbell, T. B., Crothers, K., Curtis, J. L., et al. (2013). Comparison of the respiratory microbiome in healthy nonsmokers and smokers. Am. J. Respir. Crit. Care Med. 187, 1067-1075. doi: 10.1164/rccm.201210-1913OC

Morton, E. R., Lynch, J., Froment, A., Lafosse, S., Heyer, E., Przeworski, M., et al. (2015). Variation in rural african gut microbiota is strongly correlated with colonization by entamoeba and subsistence. PLoS Genet. 11:e1005658. doi: 10.1371/journal.pgen.1005658

Nam, Y. D., Jung, M. J., Roh, S. W., Kim, M. S., and Bae, J. W. (2011). Comparative analysis of Korean human gut microbiota by barcoded pyrosequencing. PLoS ONE 6:e22109. doi: 10.1371/journal.pone.0022109

Nasidze, I., Li, J., Quinque, D., Tang, K., and Stoneking, M. (2009). Global diversity in the human salivary microbiome. Genome Res. 19, 636-643. doi: 10.1101/gr.084616.108

Nasidze, I., Li, J., Schroeder, R., Creasey, J. L., Li, M., and Stoneking, M. (2011). High diversity of the saliva microbiome in Batwa Pygmies. PLoS ONE 6:e23352. doi: 10.1371/journal.pone.0023352

Nishijima, S., Suda, W., Oshima, K., Kim, S. W., Hirose, Y., Morita, H., et al. (2016). The gut microbiome of healthy Japanese and its microbial and functional uniqueness. DNA Res. 23, 125-133. doi: 10.1093/dnares/dsw002

Noble, W. C. (1984). Skin microbiology: coming of age. J. Med. Microbiol. 17, 1-12. doi: 10.1099/00222615-17-1-1

Nomura, I., Gao, B., Boguniewicz, M., Darst, M. A., Travers, J. B., and Leung, D. Y. (2003a). Distinct patterns of gene expression in the skin lesions of atopic dermatitis and psoriasis: a gene microarray analysis. J. Allergy Clin. Immunol. 112, 1195-1202. doi: 10.1016/j.jaci.2003.08.049

Nomura, I., Goleva, E., Howell, M. D., Hamid, Q. A., Ong, P. Y., Hall, C. F., et al. (2003b). Cytokine milieu of atopic dermatitis, as compared to psoriasis, skin prevents induction of innate immune response genes. J. Immunol. 171, 3262-3269. doi: 10.4049/jimmunol.171.6.3262

Obregon-Tito, A. J., Tito, R. Y., Metcalf, J., Sankaranarayanan, K., Clemente, J. C., Ursell, L. K., et al. (2015). Subsistence strategies in traditional societies distinguish gut microbiomes. Nat. Commun. 6:6505. doi: 10.1038/ncomms7505

O’Keefe, S. J., Chung, D., Mahmoud, N., Sepulveda, A. R., Manafe, M., Arch, J., et al. (2007). Why do African Americans get more colon cancer than Native Africans? J. Nutr. 137, 175S-182S.

O’Keefe, S. J., Li, J. V., Lahti, L., Ou, J., Carbonero, F., Mohammed, K., et al. (2015). Fat, fibre and cancer risk in African Americans and rural Africans. Nat. Commun. 6:6342. doi: 10.1038/ncomms7342

Ong, P. Y., Ohtake, T., Brandt, C., Strickland, I., Boguniewicz, M., Ganz, T., et al. (2002). Endogenous antimicrobial peptides and skin infections in atopic dermatitis. N. Engl. J. Med. 347, 1151-1160. doi: 10.1056/NEJMoa021481

Ou, J., Carbonero, F., Zoetendal, E. G., DeLany, J. P., Wang, M., Newton, K., et al. (2013). Diet, microbiota, and microbial metabolites in colon cancer risk in rural Africans and African Americans. Am. J. Clin. Nutr. 98, 111-120. doi: 10.3945/ajcn.112.056689

Peek, R. M. Jr., and Blaser, M. J. (2002). Helicobacter pylori and gastrointestinal tract adenocarcinomas. Nat. Rev. Cancer 2, 28-37. doi: 10.1038/nrc703

Qin, J., Li, R., Raes, J., Arumugam, M., Burgdorf, K. S., Manichanh, C., et al. (2010). A human gut microbial gene catalogue established by metagenomic sequencing. Nature 464, 59-65. doi: 10.1038/nature08821

Qin, J., Li, Y., Cai, Z., Li, S., Zhu, J., Zhang, F., et al. (2012). A metagenomewide association study of gut microbiota in type 2 diabetes. Nature 490, 55-60. doi: $10.1038 /$ nature 11450

Rampelli, S., Schnorr, S. L., Consolandi, C., Turroni, S., Severgnini, M., Peano, P., et al. (2015). Metagenome sequencing of the hadza hunter-gatherer gut microbiota. Curr. Biol. 25, 1682-1693. doi: 10.1016/j.cub.2015.04.055

Ravel, J., Gajer, P., Abdo, Z., Schneider, G. M., Koenig, S. S., McCulle, S. L., et al. (2011). Vaginal microbiome of reproductive-age women. Proc. Natl. Acad. Sci. U.S.A. 108(Suppl. 1), 4680-4687. doi: 10.1073/pnas.1002611107

Rehman, A., Rausch, P., Wang, J., Skieceviciene, J., Kiudelis, G., Bhagalia, K., et al. (2016). Geographical patterns of the standing and active human gut microbiome in health and IBD. Gut 65, 238-248. doi: 10.1136/gutjnl-2014-308341

Roth, R. R., and James, W. D. (1989). Microbiology of the skin: resident flora, ecology, infection. J. Am. Acad. Dermatol. 20, 367-390. doi: 10.1016/S0190-9622(89)70048-7

Sankaranarayanan, K., Ozga, A. T., Warinner, C., Tito, R. Y., Obregon-Tito, A. J., Xu, J., et al. (2015). Gut microbiome diversity among cheyenne and arapaho individuals from Western Oklahoma. Curr. Biol. 25, 3161-3169. doi: 10.1016/j.cub.2015.10.060

Schnorr, S. L., Candela, M., Rampelli, S., Centanni, M., Consolandi, C., Basaglia, G., et al. (2014). Gut microbiome of the Hadza hunter-gatherers. Nat. Commun. 5:3654. doi: $10.1038 /$ ncomms4654

Stearns, J. C., Davidson, C. J., McKeon, S., Whelan, F. J., Fontes, M. E., Schryvers, A. B., et al. (2015). Culture and molecular-based profiles show shifts in bacterial communities of the upper respiratory tract that occur with age. ISME J. 9, 1246-1259. doi: 10.1038/ismej.2014.250

Tagami, H. (2008). Location-related differences in structure and function of the stratum corneum with special emphasis on those of the facial skin. Int. J. Cosmet. Sci. 30, 413-434. doi: 10.1111/j.1468-2494.2008.00459.x

Tana, C., Umesaki, Y., Imaoka, A., Handa, T., Kanazawa, M., and Fukudo, S. (2010). Altered profiles of intestinal microbiota and organic acids may be the origin of symptoms in irritable bowel syndrome. Neurogastroenterol. Motil. 22, e114-e515. doi: 10.1111/j.1365-2982.2009.01427.x

Tarabichi, Y., Li, K., Hu, S., Nguyen, C., Wang, X., Elashoff, D., et al. (2015). The administration of intranasal live attenuated influenza vaccine induces changes in the nasal microbiota and nasal epithelium gene expression profiles. Microbiome 3:74. doi: 10.1186/s40168-015-0133-2

Turnbaugh, P. J., Ley, R. E., Hamady, M., Fraser-Liggett, C. M., Knight, R., and Gordon, J. I. (2007). The human microbiome project. Nature 449, 804-810. doi: $10.1038 /$ nature06244

Turnbaugh, P. J., Ley, R. E., Mahowald, M. A., Magrini, V., Mardis, E. R., and Gordon, J. I. (2006). An obesity-associated gut microbiome with increased capacity for energy harvest. Nature 444, 1027-1031. doi: 10.1038/nature05414 
Tyakht, A. V., Kostryukova, E. S., Popenko, A. S., Belenikin, M. S., Pavlenko, A. V., Larin, A. K., et al. (2013). Human gut microbiota community structures in urban and rural populations in Russia. Nat. Commun. 4:2469. doi: 10.1038 /ncomms3469

Van Treuren, W., Ponnusamy, L., Brinkerhoff, R. J., Gonzalez, A., Parobek, C. M., Juliano, J. J., et al. (2015). Variation in the microbiota of ixodes ticks with regard to geography, species, and sex. Appl. Environ. Microbiol. 81, 6200-6209. doi: 10.1128/AEM.01562-15

Vebo, H. C., Karlsson, M. K., Avershina, E., Finnby, L., and Rudi, K. (2016). Bead-beating artefacts in the Bacteroidetes to Firmicutes ratio of the human stool metagenome. J. Microbiol. Methods 129, 78-80. doi: 10.1016/j.mimet.2016.08.005

Verstraelen, H., Vilchez-Vargas, R., Desimpel, F., Jauregui, R., Vankeirsbilck, N., Weyers, S., et al. (2016). Characterisation of the human uterine microbiome in non-pregnant women through deep sequencing of the V1-2 region of the 16S rRNA gene. PeerJ 4:e1602. doi: 10.7717/peerj. 1602

Wade, W. G. (2013). The oral microbiome in health and disease. Pharmacol. Res. 69, 137-143. doi: 10.1016/j.phrs.2012.11.006

Walker, A. W., Martin, J. C., Scott, P., Parkhill, J., Flint, H. J., and Scott, K. P. (2015). 16S rRNA gene-based profiling of the human infant gut microbiota is strongly influenced by sample processing and PCR primer choice. Microbiome 3:26. doi: 10.1186/s40168-015-0087-4

Wang, Z., Klipfell, E., Bennett, B. J., Koeth, R., Levison, B. S., Dugar, B., et al. (2011). Gut flora metabolism of phosphatidylcholine promotes cardiovascular disease. Nature 472, 57-63. doi: 10.1038/nature09922

Yap, G. C., Chee, K. K., Hong, P. Y., Lay, C., Satria, C. D., Sumadiono, S., et al. (2011). Evaluation of stool microbiota signatures in two cohorts of Asian (Singapore and Indonesia) newborns at risk of atopy. BMC Microbiol. 11:193. doi: 10.1186/1471-2180-11-193

Yatsunenko, T., Rey, F. E., Manary, M. J., Trehan, I., Dominguez-Bello, M. G., Contreras, M., et al. (2012). Human gut microbiome viewed across age and geography. Nature 486, 222-227. doi: 10.1038/nature11053
Yi, H., Yong, D., Lee, K., Cho, Y. J., and Chun, J. (2014). Profiling bacterial community in upper respiratory tracts. BMC Infect. Dis. 14:583. doi: 10.1186/s12879-014-0583-3

Ying, S., Zeng, D. N., Chi, L., Tan, Y., Galzote, C., Cardona, C., et al. (2015). The influence of age and gender on skin-associated microbial communities in urban and rural human populations. PLoS ONE 10:e0141842. doi: 10.1371/journal.pone.0141842

Zeller, G., Tap, J., Voigt, A. Y., Sunagawa, S., Kultima, J. R., Costea, P. I., et al. (2014). Potential of fecal microbiota for early-stage detection of colorectal cancer. Mol. Syst. Biol. 10:766. doi: 10.15252/msb.20145645

Zhernakova, A., Kurilshikov, A., Bonder, M. J., Tigchelaar, E. F., Schirmer, M., Vatanen, T., et al. (2016). Population-based metagenomics analysis reveals markers for gut microbiome composition and diversity. Science 352, 565-569. doi: 10.1126/science.aad3369

Zhou, X., Brown, C. J., Abdo, Z., Davis, C. C., Hansmann, M. A., Joyce, P., et al. (2007). Differences in the composition of vaginal microbial communities found in healthy Caucasian and black women. ISME J. 1:121-133. doi: 10.1038 /ismej.2007.12

Zhou, X., Hansmann, M. A., Davis, C. C., Suzuki, H., Brown, C. J., Schutte, U., et al. (2010). The vaginal bacterial communities of Japanese women resemble those of women in other racial groups. FEMS Immunol. Med. Microbiol. 58, 169-181. doi: 10.1111/j.1574-695X.2009.00618.x

Conflict of Interest Statement: The authors declare that the research was conducted in the absence of any commercial or financial relationships that could be construed as a potential conflict of interest.

Copyright (c) 2017 Gupta, Paul and Dutta. This is an open-access article distributed under the terms of the Creative Commons Attribution License (CC BY). The use distribution or reproduction in other forums is permitted, provided the original author(s) or licensor are credited and that the original publication in this journal is cited, in accordance with accepted academic practice. No use, distribution or reproduction is permitted which does not comply with these terms. 